\title{
A LANDMARK EXTRACTION METHOD ASSOCIATED WITH GEOMETRIC FEATURES AND LOCATION DISTRIBUTION
}

\author{
Zhang Wenjing, Li Jiatian, Wang Yu, Xiao Yi, Liu Peng, Zhang Sijia* \\ Institute of land and Resources Engineering, Kunming University of Science and Technology, Kunming, China - \\ 2248853762@qq.com
}

KEY WORDS: Landmark Extraction, Geometric Features, Crevice, Horizon Segmentation, Voronoi Diagram

\begin{abstract}
:
Landmark plays an important role in spatial cognition and spatial knowledge organization. Significance measuring model is the main method of landmark extraction. It is difficult to take account of the spatial distribution pattern of landmarks because that the significance of landmark is built in one-dimensional space. In this paper, we start with the geometric features of the ground object, an extraction method based on the target height, target gap and field of view is proposed. According to the influence region of Voronoi Diagram, the description of target gap is established to the geometric representation of the distribution of adjacent targets. Then, segmentation process of the visual domain of Voronoi $\mathrm{K}$ order adjacent is given to set up target view under the multi view; finally, through three kinds of weighted geometric features, the landmarks are identified. Comparative experiments show that this method has a certain coincidence degree with the results of traditional significance measuring model, which verifies the effectiveness and reliability of the method and reduces the complexity of landmark extraction process without losing the reference value of landmark.
\end{abstract}

\section{INTRODUCTION}

Landmark is a spatial form that can be recognized by the cognitive population in a certain space and can be a symbol in both psychological and geographical significance. The landmark is easily identified and remembered from many directions in the geographical space. It is a prominent element in the space. In spatial cognition, organization and expression in the process of landmarks as a reference to organize and express the spatial knowledge (Gong et al., 2016a). In the process of spatial location description, it can be used to help describe the spatial location of the target and express spatial information. Based on path selection and navigation, landmarks can effectively help us to understand the structured environment, and gradually establish the path and structure knowledge of the environment (Zhang et al., 2012b; Li et al., 2014a; Zhang et al., 2013a). Therefore, the method of landmark extraction has always been a hot issue in the field of geographic information science.

At present, the main way of landmark extraction is to build significance model based on related factors, and achieve the purpose of extracting landmarks by calculating the significance of land objects. Sorrows (1997a) defines the landmark as a distinct discernible element independent of any observer, he thinks that the landmark has 3 aspects of significance, visual, structural and semantic. The ground object with strong visual features, unique semantics and important space position can become a landmark. For this reason, some scholars have proposed and developed a significance measuring model consisting of these three indicators. Raubal and Winter (2002a) construct significant measuring models from three aspects: visual attractiveness, semantic attractiveness and structural attractiveness. Due to the different environment and user needs, more factors are needed to consider the significance of objects. Caduff (2009a) and Timpf put forward a more complex saliency measuring model, which includes three significant vectors and a series of member variables, including perception, cognition and scene. And Bayesian network is used to characterize the causality between spatial objects, observers and the environment. In order to acquire hierarchical spatial knowledge that can be used for intelligent path guidance, (Zhao et al., 2011a) analyze the factors that affect the significance of POI from three aspects, public cognition, spatial distribution and individual characteristics, and construct the POI significance measuring model using three indicators, including public awareness, city centrality and characteristic attribute value. In addition, to obtain urban spatial knowledge for intelligent location service in time and accurately, (Wang et al., 2016a), have discussed the factors that influence the POI significance from the number of check-in data, the number of check-in users and user influence factors, and then propose a POI significance measuring model based on the position checkin data. The core of the significance measuring is to choose important factors, put in the linear space, and then the factors are classified and weighted to be used for the final evaluation. The whole process ignores the spatial distribution of objects, making the sensitivity of the extracted landmark to the spatial distribution is low and the correlation is not strong.

The target individual of the ground object and its adjacent target influence each other, and the geometric shape of the ground object and the distribution of the adjacent target are the main factors that form the landmark ( $\mathrm{Li}$ et al., 2003a; Zheng et al., 2008a; Tao et al., 2010a). In this paper, we use the target height as the basis of geometric form, and construct the goal gap in the scope of the plane Voronoi Diagram, which is used to describe the interaction between targets. In order to reflect the spatial distribution of ground objects more accurately, we take Voronoi $\mathrm{K}$ neighborhood as the sphere of action, and divide it into multiple neighborhoods to simulate the visibility of objects in multiple directions. In order to achieve the purpose of rapid extraction of landmarks, the target height, target gap and significant influence factors are weighted to construct significance model, and the significance are measured from the

* Corresponding author: Li Jiatian (ljtwcx@163.com) 
visibility of multiple directions to realize the target extraction, which reduces the complexity and the extraction results under spatial cognition.

\section{GEOMETRIC FEATURES AND POSITION DISTRIBUTION FACTORS}

\subsection{Geometric feature selection}

Voronoi Diagram is a basic geometric structure of space subdivision, it is shown as a group of growing sources expanding to the surrounding area, until the meet, to form the collection of the sphere of influence of the growing source (Gold, 1997a; Chen et al., 2003a). Voronoi Diagram contains many excellent spatial general nature, such as proximity and sphere of influence (Chen et al., 2001a; Chen et al., 2004a). It is considered as a powerful tool to study and solve the spatial relationship and spatial analysis of the visual field of Geographic Information Science, and to optimize the space configuration and other related problems (Liu et al., 2004a; Li et al., 2016a).

Definition 1 General Voronoi Diagram: Suppose that point set $\boldsymbol{P}=\left\{p_{1}\left(x_{1}, y_{1}\right), \ldots, p_{n}\left(x_{n}, y_{n}\right)\right\} \subset \boldsymbol{R}^{2}, 1<n<\infty, p$ is an arbitrary point, $p(x, y) \in \boldsymbol{R}^{2}$,point $p_{i}\left(x_{i}, y_{i}\right)$ and $p_{j}\left(x_{j}, y_{j}\right)$ are existed, the region expressed in (1) is the Voronoi region of $p_{i}$ :

$$
\operatorname{vor}\left(p_{i}\right)=\left\{\left\|p_{i}-p\right\| \leq\left\|p_{j}-p\right\|, i \neq j, i, j \in N\right\}
$$

where $\left\|p_{i}-p\right\|=\sqrt{\left(x_{i}-x\right)^{2}+\left(y_{i}-y\right)^{2}}$. General Voronoi Diagram is expressed as follow:

$$
V=\left\{\operatorname{vor}\left(p_{1}\right), \cdots, \operatorname{vor}\left(p_{n}\right)\right\}
$$

There are two kinds of proximity between the space targets, Euclidean distance proximity and Voronoi proximity. Compared with Euclidean distance proximity, Voronoi proximity has more advantages in measuring and neighboring target distribution.

Definition 2 Voronoi proximity: If the space target set $\boldsymbol{O}=\left\{o_{1}, o_{2}\right.$, $\left.o_{3}, \ldots, o_{n}\right\} \in \boldsymbol{R}^{2}$ is existed, $1<n<\infty$, for any given $o_{i}, o_{j},(i \neq j), \operatorname{vor}\left(o_{i}\right)$ and $\operatorname{vor}\left(o_{j}\right)$ represent the Voronoi regions of the target $\mathrm{o}_{i}$ and $o_{j}$, respectively. If formula (3) is established:

$$
\operatorname{vor}\left(o_{i}\right) \cap \operatorname{vor}\left(o_{j}\right) \neq \varnothing
$$

then the target $o_{i}$ and $o_{j}$ are adjacent to Voronoi.

Definition 3 Voronoi $k$ order adjacency: If the space target set $\boldsymbol{O}=\left\{o_{1}, o_{2}, o_{3}, \ldots, o_{n}\right\} \in \boldsymbol{R}^{2}$ is exsited, $1<n<\infty$, for any given $o_{i}, o_{j}$, $(i \neq j)$, if the target $o_{i}$ reaches the target $o_{j}$ through the minimum Voronoi neighbor step number $k, o_{i}$ is adjacent to $o_{j}$ of the Voronoi $k$ order.

The significance of landmarks is mainly reflected in their appearance, structure or semantics differences, compared with other spatial objects in the surrounding environment, including the shape, size, location, roles and cultural connotations, etc. In a strange environment, without considering the semantics of special features and the structure factors, the large and high ground objects are often leave the first impression on the observers in the visible range because of their visual attractiveness and strong reference. In Voronoi diagram, the size of the space occupied by the ground can be quantitatively described by its sphere of influence, so the area and height of the surface area are taken as the geometric characteristics of the quantitative description of landmarks.

\subsection{Gap describe}

Although, to a certain extent, sphere of influence of Voronoi Diagram is determined by the area of the source of growth, it is also influenced by the intensity of the ground objects. It is not suitable to select the surface area directly as the geometric feature of the quantitative description of the landmark. In the actual situation, for some land surface objects which are not prominent in the area and height, the central buildings of some squares can still be selected as landmarks. Therefore, the density of ground objects in the space range is considered, according to the proximity relation of Voronoi, the area distribution of the Voronoi area and the spatial correlation (Kang et al., 2017a; Zhang et al., 2014a; Li et al., 2014a; Li et al., 2016a). The larger the ratio of the area of the ground to the area of the Voronoi, the more aggregated the spatial distribution of the ground objects is. It is assumed that the total area of ground objects in the space range is $S$, and the total area of the ground object Voronoi is $S_{v o r}$, then the degree of ground density is expressed as:

$$
\rho=\frac{S}{S_{\text {vor }}}
$$

For a single object in the space range, its area is expressed as $s^{\prime}$, the corresponding area of Voronoi region is $s_{v o r}^{\prime}$, then the target gap can be described as:

$$
q=\frac{s^{\prime}-s_{v o r}^{\prime}}{s_{v o r}^{\prime}}
$$

In order to describe the gap between a single object target and its adjacent target set, all the adjacent objects of the $k$ order of the ground object are selected as the adjacent target set.

$$
Q=q+\sum_{i=1}^{k} \sum_{j=1}^{n} q_{i}
$$

where $q_{i}$ represents a $k$ order adjacent to the ground object, and the number of adjacent objects adjacent to the $k$ order of the ground object is n. $Q$ is used to measure the size of the gap between the object and the adjacent target set. Many buildings have some dependency relationship with others in the space range, in order to make the description of the gap more conform to the actual situation, the computation of gap can be extended to $k(k>1)$ order adjacency of the ground object.

\subsection{Segmentation of the Horizon}

When the spatial cognition is carried out from different perspectives, there will be some targets that are too large or too high in a certain direction, and many objects are shielded from each other within the visual angle of the human eye. The difference in the visibility of the objects in different directions makes the spatial cognition incomplete. For this reason, considering the strong correlation between the objects in the view area, we divide the spatial scope into multiple viewable areas, and simulate the visibility of objects under multiple views in a two-dimensional plane with multiple viewports.

Because the Voronoi regions in the plane space do not overlap each other, and cover the whole two-dimensional space continuously, the continuous coverage links the spatial objects, implicitly expressing the neighborhood information between the spatial growth targets. Therefore, the segmentation of visual domain segmentation is carried out according to the proximity relation of the Voronoi $k$ order. Assuming that the number of Voronoi 1 order adjacent objects is $\mathrm{m}$, based on the $\mathrm{m}$ one order adjacent objects, the local spatial scope centered on the target surface is divided into $\mathrm{m}$ visual fields. As shown in Figure 1, the 
yellow part is the target of the ground, and the number of the adjacent objects in the 1 order of Voronoi is 7. Taking the object as the center, 7 viewable areas are divided, where the direction of the arrow is the growth direction of the visible domain. The $m$ visual domains of the target object can be expressed as:

$$
\operatorname{Reg}=\left\{\operatorname{Seg}_{1}, \operatorname{Seg}_{2}, \cdots, \operatorname{Seg}_{m}\right\}
$$

in which $\mathrm{Seg}_{i}$ represents a field of view centered on the ground object.

According to Voronoi $k$ order adjacency relation, the Voronoi 1 order adjacent objects of target objects can be regarded as the first layer of visible objects. After $k$ segmentations, the visible domain can be expressed as:

$$
\text { Seg }=\left\{O_{i}, i=0,1, \cdots, k\right\}
$$

where $O_{i}(1 \leqslant i \leqslant k)$ represents the collection of objects in the field of view of layer $i$.

The adjacent objects of the Voronoi 1 order of the $i(i \geqslant 1)$ layer of the visual domain are expressed as:

$$
G=\left\{g_{i}, i=0,1, \cdots, n\right\}
$$

The adjacent objects of the ground object of Voronoi $k(k \geqslant 2)$ order are described as:

$$
G^{k}=\left\{G_{i}^{k}, i=0,1, \cdots, l\right\}
$$

The overlapped part of the adjacent object of the Voronoi 1 order and the Voronoi $k$ order of the object of the $i$ layer of the visual domain is regarded as the $i+1$ layer of the visual domain here, which can be expressed as:

$$
O_{i}=\left\{G \cap G^{k}\right\}=\left\{\begin{array}{cc}
\left\{g_{i}, i=0,1,\right. & \cdots, n\} \cap\left\{G_{i}^{k}, \quad i=\right. \\
0,1, & \cdots, m\}\}
\end{array}\right.
$$

As shown in Figure 2, the red part is the first floor of the visual field after two divisions, and the blue part is the second layer.

The visual domain segmentation process is described as follows: 1. Find the number of adjacent objects of Voronoi 1 order is $m$, then the number of the field of view of the ground object is $\mathrm{m}$, and the adjacent objects of Voronoi 1 order are regarded as the objects of the first visible objects.

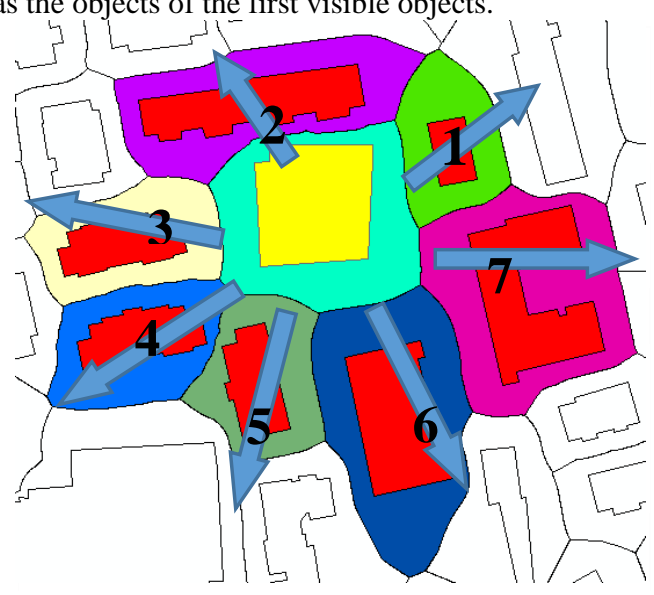

Figure .1 The first order adjacent ground objects of the ground object
2. Find the adjacent ground object set $G$ of Voronoi 1 order of the $i(i \geqslant 1)$ layer and the adjacent ground object set $G_{k}$ of Voronoi $k(k \geqslant 2)$ order, then the grounds of layer $i+1$ is looked up according to (5).

3. $i++, k++$, repeat step 2 .

4. The next segmentation of the visual domain is carried out, and repeat step 2 and 3 .

5. Repeat step 4, until all the visual domains of ground objects are segmented. The result of the visible domain segmentation of a ground object is shown in Figure 3.

\subsection{Extracting process}

In this paper, a significant model is constructed to calculate the saliency of the objects in the visual domain. To unify the gaps in all the objects in the visual field to a single scale, $\rho$ is taken as the scale factor, it is also necessary to standardize the significant influence factors, and the original data are transformed into interval $[0,1]$, using the method of extreme standardization, the unified gap is expressed as:

$$
Q^{\prime}=\rho \cdot Q
$$

The significance model can be expressed as:

$$
f(X)=w_{1} \cdot h+w_{2} \cdot Q^{\prime}+w_{3} \cdot m
$$

where $w_{1}, w_{2}$ and $w_{3}$ respectively represent are the weights of the height and gap of the ground and the number of visual fields.

When using the significance model to extract the landmark, it is extracted from the single visual domain of the object separately. After all the visual fields of the objects are traversed, all the objects in the area are traversed. In order to make the selected landmark more valuable, the spatial correlation of the extracted landmark objects is enhanced. After traversing all the visible domains of objects, we merge the adjacent visual domains on both sides of a visual field as a whole to evaluate the saliency of objects. As shown in Figure 4, the blue region represents the result of the combination of the two visual domains adjacent. After merging, the scope of the visual field is expanded, which is equal to the expansion of the visual angle. And the merged visual domain can be expressed as:

$$
O_{c}=O_{i-1} \cup O_{i} \cup O_{i+1}
$$

The target gap in the visual domain $O_{c}$ is recalculated, and (8) is

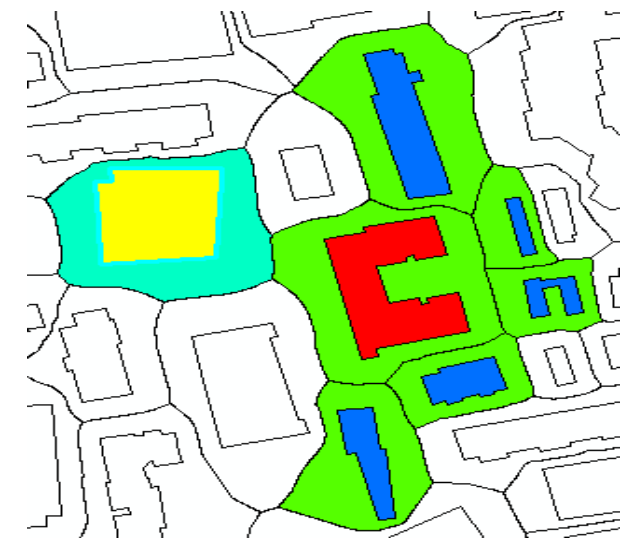

Figure .2 Two segmentation schematic of a single visual field 
used to measure all the objects in the visual field, and the threshold value of the landmark selection is constant before and after the merger. The set of ground objects that can be used as landmark in the three visual fields before merging is $L\left\{g_{1}\right.$, $\left.g_{2}, \ldots, g_{n}\right\}$, and after the merging, the set is $L^{\prime}\left\{g^{\prime}, g_{2}^{\prime}, \ldots, g_{m}^{\prime}\right\}$, then the ground objects that are landmarks are intersection of the two

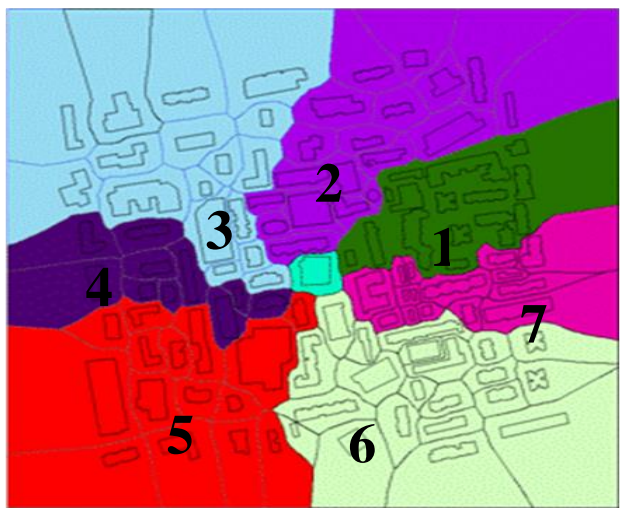

Figure 3. The result of visual domain segmentation

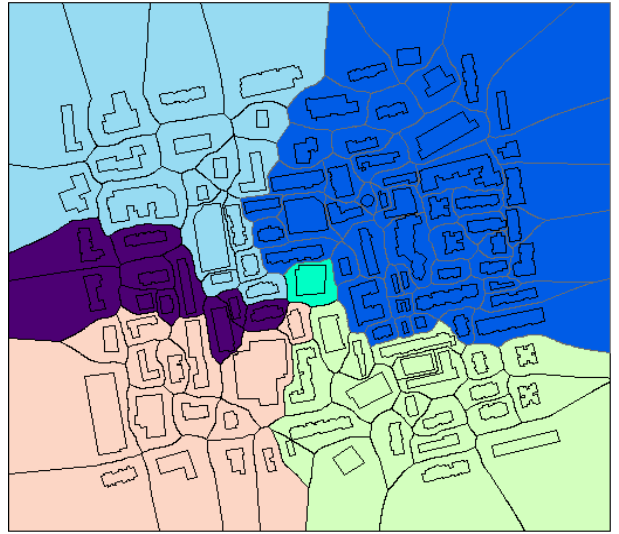

Figure 4. Contiguous visual domain merging result schematic sets, which can be expressed as:

$$
L_{M}=L \cap L^{\prime}
$$

$L_{M}$ is the final result.

\section{EXPERIMENTS AND ANALYSES}

In order to verify the feasibility and effectiveness of this method, all the objects in the experimental area are segmented by the visual domain. Then the landmark is extracted from the visual domain of the single ground object and the result is compared with the proposed method in previous work (Kim, 2017a). The validity of this method is verified by the coincidence degree of the two methods.

The experiment selected part of the vector data in the No.2 Ring of Kunming city of Yunnan province, the main source of the extraction of this landmark is a plane-like object, a small number of spot objects are not considered, the experimental data include 1918 surface objects. The two-dimensional space position coordinate and the attribute information of the plane-like objects are obtained as the input mode, the map database includes planelike objects and the notes of them. The construction of Voronoi Diagram in the experimental area mainly decomposes the growing sources, and the plane-like object is decomposed into a point element according to the length of a certain interval. Then the boundary point is encrypted, Voronoi Diagram that is used to build polygon is converted to Voronoi Diagram that is used to build point ( $\mathrm{Li}$ et al., 2015a).

In previous work (Kim, 2017a), objects are classified through a large number of description corpus, a description from web page extraction landmark model is presented, a model for extracting roadmap from web site description is proposed, reference significance, familiarity significance and intermediary significance are considered as impact factors to build a measuring model. The weight allocation is shown in Table 1 . The results of this experiment are shown in Figure 5(a), and the results extracted by the contrast method are shown in Figure 5(b).

\begin{tabular}{ccc}
\hline Extraction method & The impact factors & Weight allocation \\
\hline \multirow{3}{*}{ This method } & Target height & 0.4 \\
& Target gap & 0.3 \\
& The number of visual ranges & 0.3 \\
\hline \multirow{3}{*}{ Contrast method } & Reference significance & 0.4 \\
& Familiarity significance & 0.3 \\
& Intermediary significance & 0.3 \\
\hline
\end{tabular}

Table 1. Two methods of impact factor and weight allocation

\begin{tabular}{cccccc}
\hline $\begin{array}{c}\text { Extraction } \\
\text { method }\end{array}$ & $\begin{array}{c}\text { Number of } \\
\text { experimental } \\
\text { objects }\end{array}$ & $\begin{array}{c}\text { Number of } \\
\text { extracted } \\
\text { landmarks }\end{array}$ & $\begin{array}{c}\text { Percentage of } \\
\text { landmarks }\end{array}$ & $\begin{array}{c}\text { Number of } \\
\text { coincident } \\
\text { landmarks }\end{array}$ & $\begin{array}{c}\text { The coincidence } \\
\text { rate of the } \\
\text { experimental } \\
\text { results of the two } \\
\text { methods }\end{array}$ \\
\hline $\begin{array}{c}\text { This } \\
\text { method }\end{array}$ & 1918 & 35 & $1.82 \%$ & 23 & $65.71 \%$ \\
$\begin{array}{c}\text { Contrast } \\
\text { method }\end{array}$ & 1918 & 42 & $2.18 \%$ & & \\
\hline
\end{tabular}

Table 2. Comparison of the experimental results of two methods 


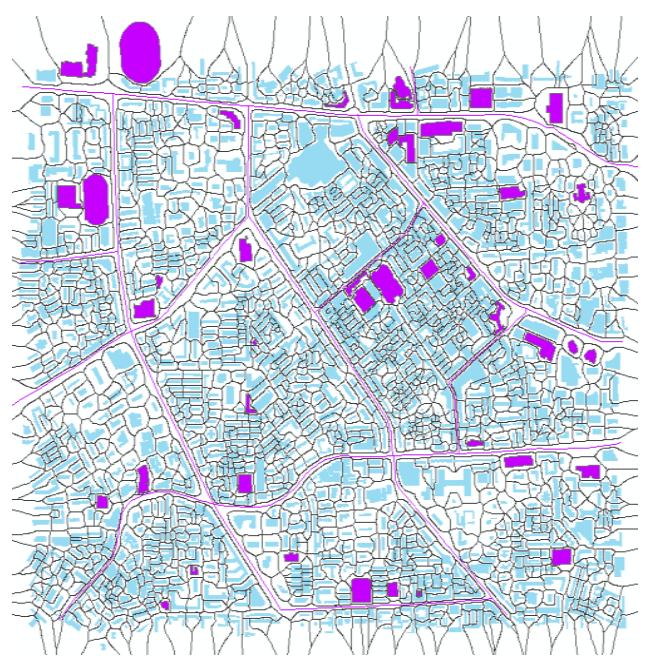

Figure(a) Result of this method

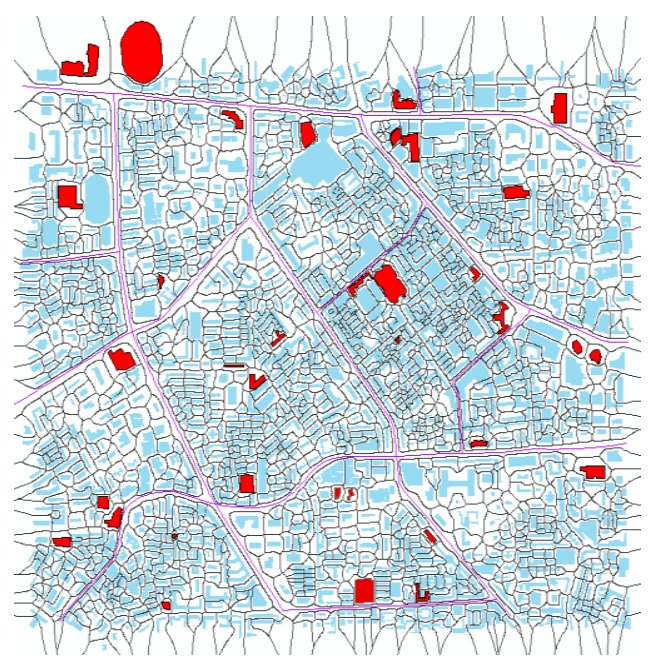

Figure(b) Result of this method

Figure 5.Extracting results of the two methods

In the experiment, the coincidence rate of the two methods varies with the range of the visual ranges. In Figure 6, the scope of the target horizons changes from 100 meters to 2000 meters, with the scope becomes larger, the coincidence rate of the results is gradually increased, and eventually reaches a stable level of $65 \%$. So, we choose the best visual range of 2000 meters.

If a ground object is extracted as a landmark in different horizons, it shows that the object is visible in many directions. Taking the extracted landmark elements as the center, the number of times that the landmark appears in different visual fields is calculated. As shown in Figure 7, the more times it appears, the more visible it is considered to be. Figure 8 shows the effect of the target gap of between the landmark and its adjacent ground objects on the visibility of the landmark. It can be seen that, for landmark with larger target gap, the distribution of its adjacent objects is relatively scattered, the correlation between the landmark and its adjacent ground objects is not strong, and its visibility is not strong. On the contrary, the landmark with smaller target gap is more concentrated in the location of its adjacent ground objects, and the landmark element has a strong correlation with its adjacent ground objects.

Although the coincidence rate is only 65.71 percent, it at least shows that this method of extracting landmark in this paper is feasible. By searching the map database, the overlapped part of the ground objects are main libraries, hospitals, commercial buildings and banks. As for the no-overlapped part, the main reasons are as follow:

(1)This paper starts with the plane Voronoi Diagram, only the height and gap of the target surface are considered as the impact factors, other feature attributes, such as color, texture, etc., are ignored. Compared with the contrast method, the method does not classify the objects in the experimental area. At the same time, the functional factors and cultural semantic factors are easily ignored, which are the main factors that cause the difference of the results;

(2)There are certain subjectivity in setting the weight of reference, familiarity, and intermediary meaning in previous work (Kim, 2017a), and the difference of the collection and the selection of network data also has an impact on the experimental results.

Compared with the existed method of building a significance measurement model, Voronoi Diagram is used to quickly select geometric features as significant influence factors, and the segmentation of visual domain is combined to realize the extraction of landmarks in this paper, which makes the method have strong timeliness, relevance and representativeness. The advantages are as follow:

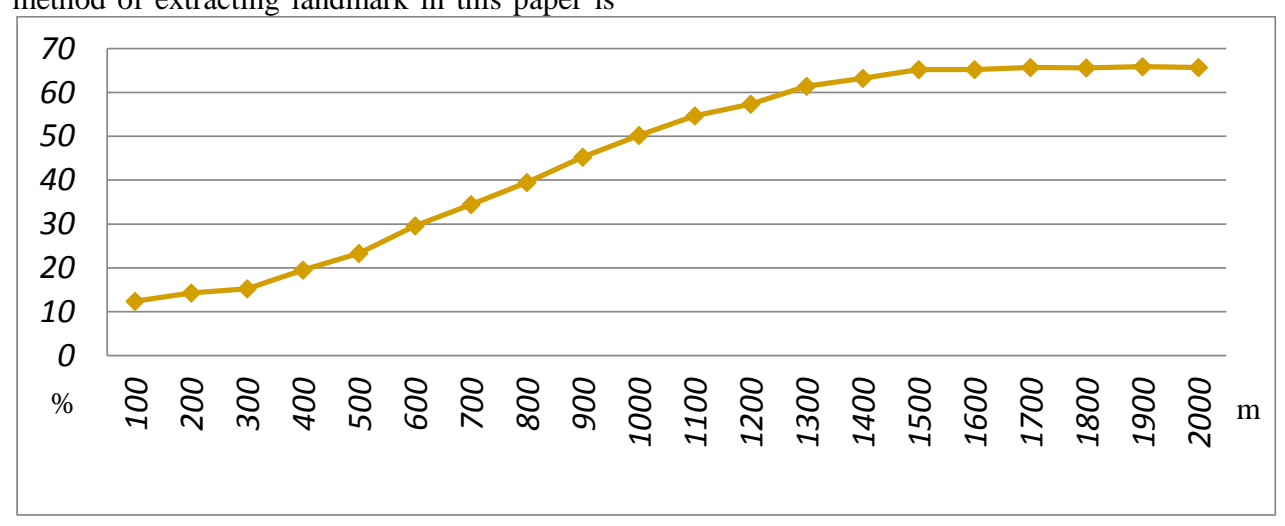

Figure6. The coincidence rate increases with the range of visibility 


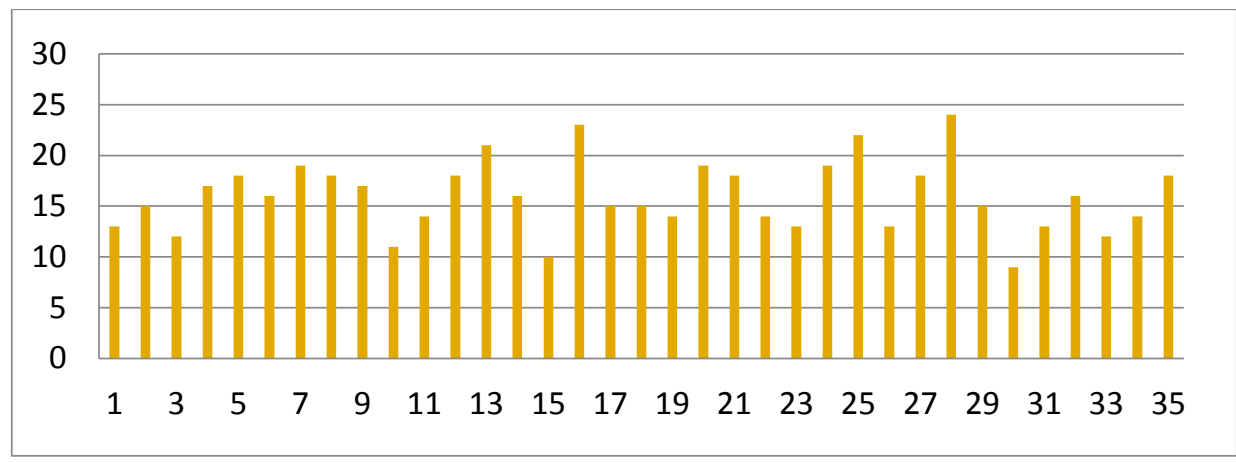

Figure 7.The visibility of the landmark in this paper

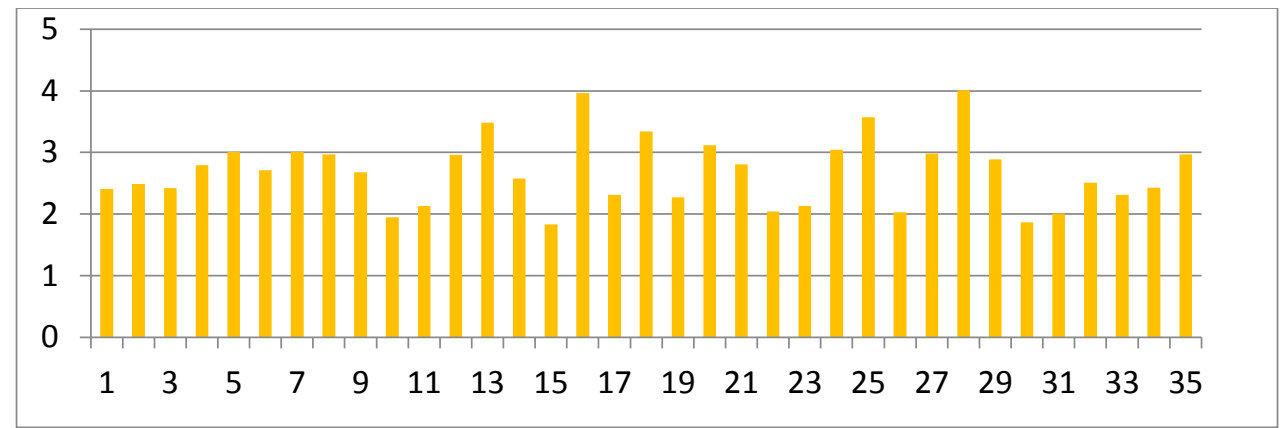

Figure 8.Gap changes of landmarks and its adjacent targets

(1)The significant influence factors are considered from the plane Voronoi Diagram, which greatly reduces the complexity of the whole process;

(2)Combined with the visibility analysis of multiple neighborhood domains, it is more consistent with the spatial cognition of multiple perspectives in the actual situation;

(3)The rapid multi-domain segmentation method makes the result more sensitive to environmental changes.

\section{CONCLUSION}

The core of this paper is to divide the spatial scope into multiple horizons based on the proximity characteristics of Voronoi Diagram, which can well meet the need that we want to cognize the space from different perspectives in real life and can adapt to environmental changes quickly. At the same time, with the help of the idea of establishing a significance measurement model by predecessors, a significance measuring model is established by selecting geometric features quickly as impact factors from Voronoi. Compared with existed methods, the complexity of the whole process can be reduced, and the results are with timeliness.

\section{ACKNOWLEDGEMENTS}

This work was partially supported by The National Natural Science Foundation of China, Nos. 41561082, 41161061.

\section{REFERENCES}

Caduff, D., and Timpf, S., 2008a. On the assessment of landmark salience for human navigation. Cognitive processing, 9(4), pp. 249-267.
Chen, Jun., Li, Chenming., and Li, Zhilin., 2001a. A VoronoiBased 9-Intersection Model for Spatial Relations. International Journal of Geographical Information Science, 15(3), pp. 201220.

Chen, Jun., Zhao Renliang., Li, Zhilin., 2004a. Voronoi-Based Korder Neighbors Relations for Spatial Analysis. ISPRS Journal of Photogrammetric and Remote Sensing. 59(1/2), pp. 60-72.

Gold, C.M., 1994a. Review: Spatial Tessellations-Concepts and Application of Voronoi Diagrams. International Journal of Geographical Information Science, 8(2), pp. 237-238.

Gong, Yongxi., Zhao, Liang., and Duan, Zhongyuan., 2016a. Hierarchical Spatial Cognition and Spatial Knowledge Organization Based on Landmarks and Voronoi Diagram. Geography and Geo-Information Science, 32(6), pp. 1-6.

Kim, J., Vasardani, M., Winter S., 2017a. Landmark Extraction from Web-Harvested Place Descriptions. KI-Künstliche Intelligenz, 31(2), pp. 151-159.

Kang, Shun., LI, Jiatian., and Wu, Hao., 2017a. An Extraction Method for Point Pattern Convergence under Voronoi Adjacency Relation. Acta Geodaetica Et Cartographica Sinica, 46(5), pp. 649-657

Li, Bijun., Fang, Zhixiang., and Ren, Juan., 2003a. Extraction of Building's Feature from Laser Scanning Data. Editorial Board of Geomatics \& Information Science of Wuhan University, 28(1), pp. $65-70$.

Li, Jiatian., Kang, Shun., and Luo, Fuli., 2014a. Point Group Generalization Method Based on Hierarchical Voronoi Diagram. In: Acta Geodaetica Et Cartographica Sinica, 43(12), pp. 13001306. 
Li, Jiatian., Kang, Shun., and Li, Xiaojuan., 2015a. Putting Model for Broad Geographic Annotation. Geomatics \& Information Science of Wuhan University, 40(1), pp. 20-25.

Li, Jiatian., LUO, Fuli., YU, Li., 2016a. The Gradient Voronoi Diagram and Construction Algorithm. Geomatics \& Information Science of Wuhan University, 41(2), pp. 163-170.

Li, Jiatian., YANG, Qili., LUO Fuli., 2015a. A Decomposition and Combination Algorithm for Voronoi Diagrams of Polylines and Polygons. Geomatics \& Information Science of Wuhan University, 40(11), pp. 1545-1550.

Liu, Jinyi,. Liu, Shuang., 2004a. A Survey on Applications of Voronoi Diagrams. Journal of Engineering Graphics, 25(2), pp. 125-132.

Li, Lin., MAO, Kai., TAN, Yongbin., 2014a. Hierarchy Landmarks Multi-granularity Description Method for Route Guidance. Acta Geodaetica Et Cartographica Sinica, 43(1), pp. 105-112.

Raubal, M., Winter, S., 2002a. Enriching wayfinding instructions with local landmarks. International conference on geographic information science. Springer, Berlin, Heidelberg, pp. 243-259.
Sorrows, M.E., Hirtle, S.C., 1997a. The nature of landmarks for real and electronic spaces, International Conference on Spatial Information Theory. Springer, Berlin, Heidelberg, pp. 37-50.

Tao, Chao., TAN, Yihua., and Cai, Huajie., et al. 2010a. Objectoriented Method of Hierarchical Urban Building Extraction from High-resolution Remote-Sensing Imagery. Acta Geodaetica Et Cartographica Sinica, 39(1), pp. 39-45.

Wang, Ming., Hu, Qingwu., and Li, Qingquan., 2016a. Extracting hierarchical landmark from check-in data. Chinese Journal of Computers, 39(2), pp. 405-413.

Zheng, Dehua., Yue, Dongjie., Yue, Jianping., 2008a. Geometric Feature Constraint Based Algorithm for Building Scanning Point Cloud Registration. Acta Geodaetica Et Cartographica Sinica, 37(4), pp. 464-468.

Zhao, Weifeng., Li, Qingquan., and Li, Bijun., 2011a. Extracting hierarchical landmarks from urban POI data. Journal of Remote Sensing, 15(5), pp. 973-988.

Zhang, Xing., Li, Qingquan., Fang, Zhixiang., 2013a. Landmark and Branch -based Pedestrian Route Complexity and Selection Algorithm. Editorial Board of Geomatics \& Information Science of Wuhan University, 38(10), pp. 1239-1242. 